
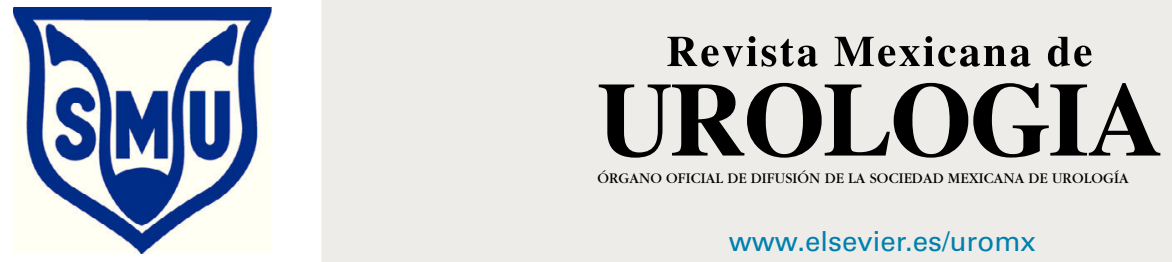

www.elsevier.es/uromx

CASO CLÍNICO

\title{
Hipogonadismo hipogonadotrópico con criptorquidia bilateral y su tratamiento laparoscópico
}

\author{
J. Padilla-Piña*, J. López Chente-Casado, A.R. García-Vásquez, \\ J.M. García de León-Gomez, J. Arriaga-Aguilar, A. Zárate-Morales, I. Gerardo-Osuna, \\ M. García-Díaz, F.E. García-Martínez y A. Razo-García
}

Servicio de Urología, Hospital General del Estado de Sonora, Universidad de Sonora (UNISON), Hermosillo, Sonora, México

Recibido el 25 de mayo de 2016; aceptado el 9 de septiembre de 2016

Disponible en Internet el 15 de noviembre de 2016

\section{PALABRAS CLAVE \\ Orquidopexia; \\ Laparoscópica; \\ Hipogonadismo; \\ Hipogonadotrófico}

\begin{abstract}
Resumen El testículo no descendido es una entidad que debe identificarse desde el nacimiento, aun más cuando esté asociado a otras malformaciones es necesario su abordaje especializado y multidisciplinario. La asociación con hipogonadismo hipogonadotrópico es inusual, con un espectro amplio de presentaciones, de acuerdo a la disfunción testicular que se presente y las consecuencias por la falta de acción de la testosterona. La laparoscopia diagnóstica y la orquidopexia laparoscópica son los tratamientos de mínima invasión de elección. Presentamos el caso de un hipogonadismo hipogonadotrópico con testículo no descendido bilateral, al cual se realizó orquidopexia laparoscópica.

(c) 2016 Sociedad Mexicana de Urología. Publicado por Masson Doyma México S.A. Este es un artículo Open Access bajo la licencia CC BY-NC-ND (http://creativecommons.org/licenses/bync-nd/4.0/).
\end{abstract}

\section{Hypogonadotropic hypogonadism with bilateral cryptorchidism and its laparoscopic treatment}

Abstract Undescended testis is an entity that should be identified from birth. Even when associated with other malformations, it requires a specialized and multidisciplinary approach. Its association with hypogonadotropic hypogonadism is unusual, with a broad spectrum of presentations according to the testicular dysfunction that presents and the consequences of the lack of testosterone action. Diagnostic laparoscopy with laparoscopic orchidopexy is the minimally invasive treatment of choice. We present herein the case of a patient with hypogonadotropic hypogonadism with bilateral undescended testis treated with laparoscopic orchidopexy.

(c) 2016 Sociedad Mexicana de Urología. Published by Masson Doyma México S.A. This is an open access article under the CC BY-NC-ND license (http://creativecommons.org/licenses/by-nc-nd/ $4.0 /)$.

\footnotetext{
* Autor para correspondencia. Cerrada Valle Cucapha \#81 Fraccionamiento Cerrada del Sol Residencial, Mexicali Baja California México. Teléfono casa: (686) 9629117, celular: (6621) 903808.

Correo electrónico: drpadillajose@gmail.com (J. Padilla-Piña).
} 


\section{Introducción}

En la actualidad, después de más de 10 años de su descripción inicial para el tratamiento de la criptorquidia, la laparoscopia es una buena alternativa en el diagnóstico y tratamiento de los testículos no palpables ${ }^{1}$.

El $10 \%$ de los recién nacidos presentan criptorquidia al nacimiento, que generalmente desciende durante el primer año de vida. En la pubertad la incidencia de criptorquidia es de $0,3 \%$ a $0,4 \%$. Es un hallazgo frecuente en muchos síndromes congénitos que conllevan hipogonadismo ${ }^{2}$.

El hipogonadismo se define como la incapacidad del testículo para realizar sus funciones: de acuerdo a la edad del sujeto producir testosterona, espermatozoides o ambos. La testosterona es una hormona que afecta a muchos tejidos del organismo. Algunos de sus efectos, como la diferenciación sexual, son tiempo dependientes y no reversibles. Otros, como sus acciones sobre la aparición y mantenimiento de los caracteres sexuales secundarios y proporciones corporales en el hombre, son parcialmente reversibles ${ }^{3}$.

El hipogonadismo se divide en primario y secundario, según la función testicular se afecte primariamente o de forma secundaria a una alteración hipotálamo-hipofisaria.

También se puede clasificar, de una forma más clínica, según el momento biológico en que se produce la insuficiencia testicular: 1) prenatal, durante la diferenciación sexual; 2) puberal, cuando el eje hipotálamo-hipófiso-testicular debe madurar y producir el desarrollo puberal; y 3) edad adulta cuando el testículo asume la producción definitiva de testosterona y espermatozoides.

Dependiendo de la edad e intensidad de la alteración de la secreción de testosterona y producción espermática la presentación clínica del hipogonadismo varía ${ }^{4}$.

El hipogonadismo masculino representa una disminución de la función testicular, con una baja producción de testosterona e infertilidad. El hipogonadismo puede deberse a un problema intrínseco de los testículos, a un fallo del eje hipotálamo-hipófisis, o a una respuesta disminuida o ausente de los órganos blancos a los andrógenos ${ }^{5}$.

La criptorquidia no tratada se asocia con infertilidad en el $70 \%$ de los casos, y se describen tumores testiculares en el $8 \%$; estos porcentajes disminuyen con el tratamiento adecuado y precoz antes de los 2-3 años ${ }^{6}$.

\section{Caso clínico}

Paciente varón de 21 años de edad, el cual tiene como antecedentes al nacimiento ausencia de pabellones auriculares e hipoacusia bilateral; requirió 4 cirugías reconstructivas de los pabellones auriculares incluyendo injerto de cartílago costal (fig. 1).

A la exploración física se observan cicatrices bilaterales en las orejas, vello facial escaso, distribución del vello genital ginecoide, micropene (fig. 2), testículo izquierdo hipotrófico localizado en el canal inguinal y el testículo derecho no palpable.

El US localiza solo el testículo izquierdo de $13 \mathrm{~mm}$ (fig. 3), se realiza gammagrama con radionúclidos en donde no fue posible localizar el testículo derecho; el perfil hormonal con FSH, LH y testosterona disminuidas.

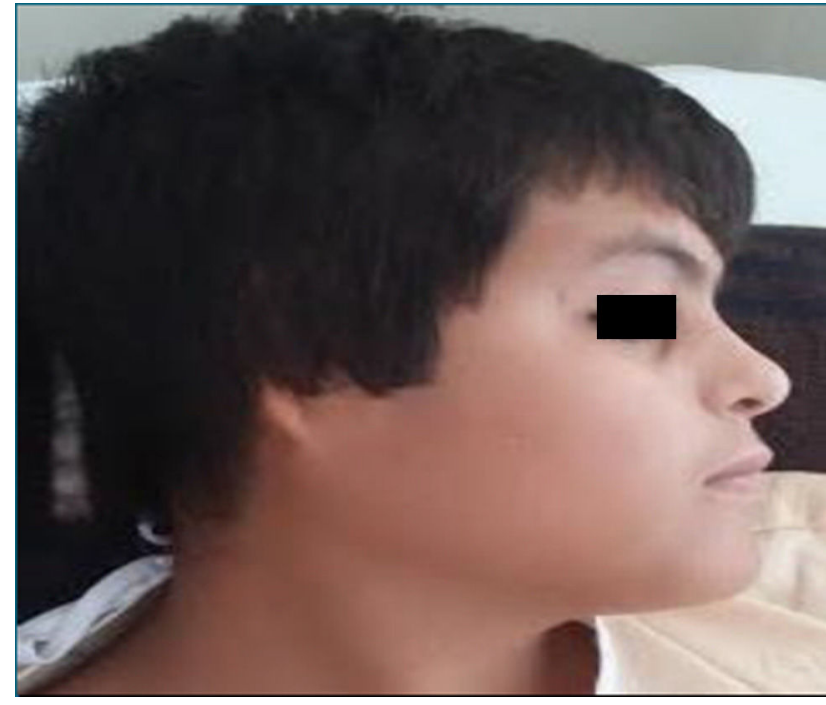

Figura 1 Perfil del paciente con ausencia de pabellones auriculares y resultados de cirugía reconstructiva con cartílago.

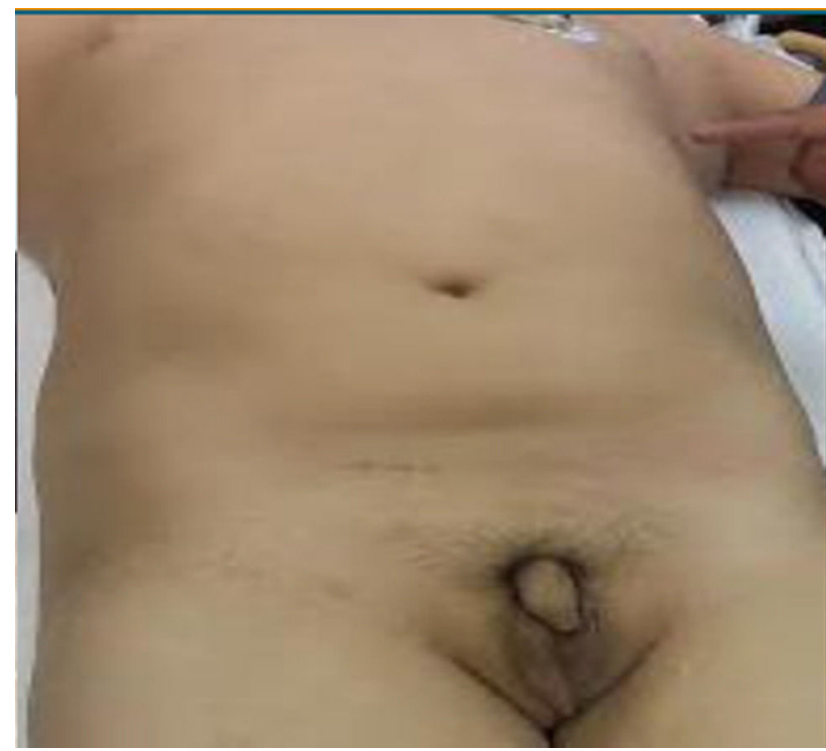

Figura 2 Imagen de micropene del paciente.

Laboratorio con biometría hemática y leucocitos $6.4, \mathrm{Hb}$ 11.6, Hto 35, plaquetas 229,000 , química sanguínea, glucosa 106, urea 55, nitrógeno ureico 25.7 , creatinina 1.6 , ácido úrico 7.7 , electrolitos séricos, sodio 142 , potasio 4.1 , cloro 106. Tiempos de coagulación, tiempo de protrombina 13.5 , tiempo parcial de tromboplastina activada 29, INR 1.0. perfil hormonal hormona folículo estimulante 0.605 , hormona luteinizante 0.101 , testosterona 0.126 .

Descripción de la técnica quirúrgica: previas valoraciones preoperatorias se procede a realizarle insición longitudinal al nivel del ombligo, y se le diseca por campos hasta la exposición de la cavidad abdominal; se le introduce el trocar con técnica de Hasson, se insufla el pneumoperitoneo con $\mathrm{CO}_{2}$ a $20 \mathrm{~mm} \mathrm{Hg}$. Este puerto se utilizará para la óptica.

Posteriormente se colocan 2 puertos en la línea media axilar de $5 \mathrm{~mm}$ con visión directa para trabajo. Se realiza laparoscopia diagnóstica encontrando un anillo inguinal 


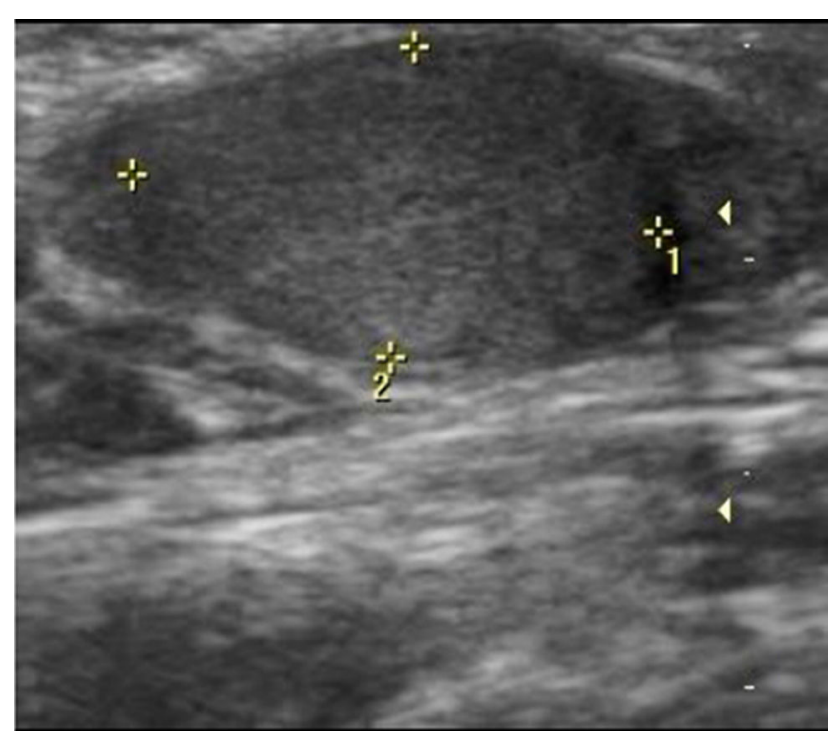

Figura 3 Identificación del testículo izquierdo de $13 \mathrm{~mm}$ por medio de ultrasonido.

ciego del lado derecho, así como testículo izquierdo al nivel del canal inguinal izquierdo, siendo el resto del abdomen normal.

En la zona inguinal los límites importantes a identificar son la arteria umbilical obliterada medialmente, los vasos gonadales externos, los vasos epigástricos superiores y la sínfisis del pubis anterior.

El triángulo formado por el conducto deferente, el testículo y los vasos gonadales guía la disección del peritoneo parietal; para despegarlo del piso de la pared lateral de la pelvis se diseca el peritoneo parietal.

Se utiliza una pinza laparoscópica fina para elevar un pliegue de peritoneo lateral a los vasos gonadales y disecar el testículo con la mayor cantidad posible de peritoneo parietal, para preservar su vascularidad, ya una vez liberado el testículo y el cordón espermático se procede a realizar una incisión del ligamento umbilical medial hasta exponer la sínfisis del pubis, la cual se puncionará con otro trocar de $5 \mathrm{~mm}$ por la parte superior para introducir una pinza laparoscópica fina desde el escroto y realizar la maniobra de Prentiss para llevar el testículo al escroto procurando hacer la tracción por el gobernaculum testis (fig. 4).

Se le realiza orquidopexia hacia el dartos con sutura absorbible, dejando los hilos de la sutura fijos a la piel del escroto (fig. 5).

Se disminuye el pneumoperitoneo y se extraen trócares bajo visión directa.

\section{Discusión}

El aparato reproductor masculino regula la diferenciación sexual, la virilización y los cambios hormonales que acompañan a la pubertad, lo que conduce finalmente a la espermatogenia y a la fertilidad. Durante el desarrollo embrionario la testosterona induce la formación del conducto de Wolff y la virilización de los genitales externos ${ }^{7}$.

En este caso es evidente el fallo testicular, ya que un testículo es evanescente y otro criptorquídico, factores que

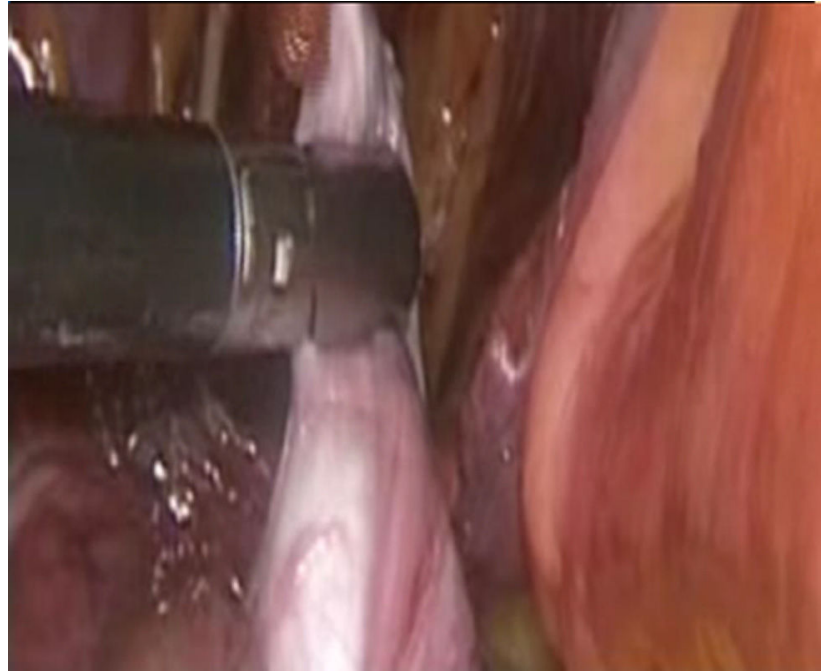

Figura 4 Maniobra de Prentiss para llevar el testículo al escroto haciendo tracción desde el gobernaculum testis.

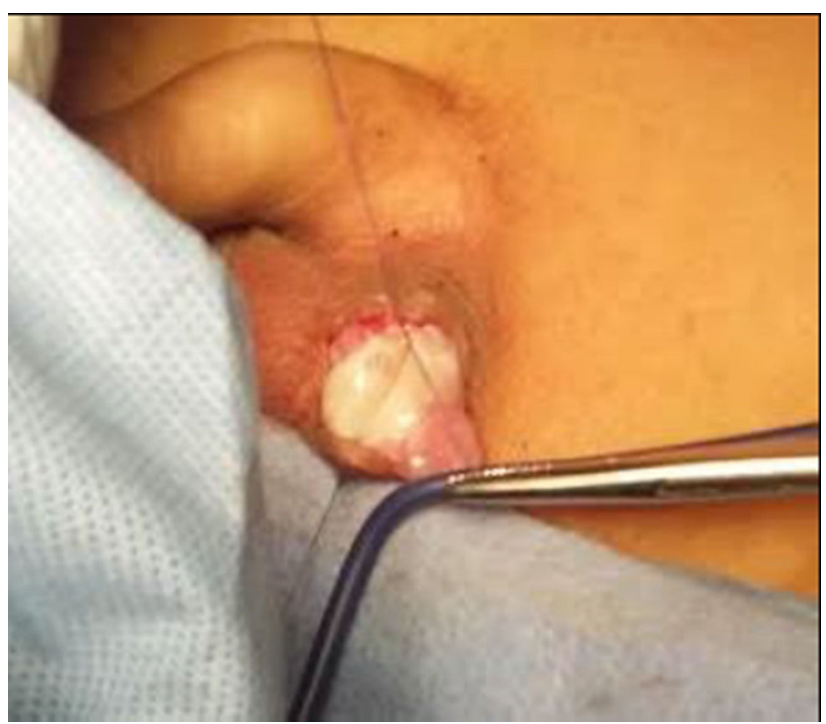

Figura 5 Orquidopexia hacia el dartos con sutura absorbible, fijando testículo a la piel del escroto.

se manifestaron en este paciente durante la pubertad; la testosterona favorece el crecimiento somático y el desarrollo de las características sexuales secundarias.

En el adulto es necesaria la testosterona para la espermatogénesis, la estimulación de la libido y el desempeño sexual normal, por lo que justifica plenamente la orquidopexia para conservar tejido testicular ${ }^{8}$.

El manejo quirúrgico de la criptorquidia ha sufrido cambios importantes en los últimos años, y ello ha permitido mejorar en grado significativo los resultados y simplificar el abordaje con la cirugía de mínima invasión ${ }^{9}$.

La orquidopexia laparoscópica ha superado a la cirugía abierta como tratamiento primario en pacientes con testículos no palpables; esto se debe en parte a que la orquidopexia laparoscópica, que permite una mayor movilización de los vasos espermáticos. 
Cada vez más la orquidopexia laparoscópica está ganando experiencia mundial, por lo que debe considerarse en la actualidad como la primera elección en el abordaje del testículo no descendido no palpable, y tal vez en el tratamiento del testículo no descendido palpable alto $^{10,11}$.

\section{Conclusión}

Presentamos el caso de un paciente varón con criptorquidia bilateral, con hipogonadismo hipogonadotrópico al que se realizó orquidopexia laparoscópica, obteniendo resultados favorables tanto en la conservación del testículo, en la búsqueda del testículo no palpable así como en la recuperación y evolución clínica del paciente con los beneficios de la laparoscopia ya probados. Consideramos la laparoscopia como la técnica de elección, tanto diagnóstica como terapéutica para los testículos no palpables.

\section{Responsabilidades éticas}

Protección de personas y animales. Los autores declaran que los procedimientos seguidos se conformaron a las normas éticas del comité de experimentación humana responsable y de acuerdo con la Asociación Médica Mundial y la Declaración de Helsinki.

Confidencialidad de los datos. Los autores declaran que han seguido los protocolos de su centro de trabajo sobre la publicación de datos de pacientes.

Derecho a la privacidad y consentimiento informado. Los autores han obtenido el consentimiento informado de los pacientes y/o sujetos referidos en el artículo. Este documento obra en poder del autor de correspondencia.

\section{Financiación}

Los autores no recibieron ningún patrocinio para llevar a cabo este artículo.

\section{Conflicto de intereses}

Los autores no tienen ningún conflicto de intereses.

\section{Bibliografía}

1. Ismail K, Ashour M, El-Afifi M, et al. Laparoscopy in the management of impalpable testis: Series of 64 cases. World J Surg. 2009;33:1514-9.

2. Geuvbashian G. Outcome of surgical management of nonpalpable testes. Urology Annals. 2013;4:273-6.

3. Virtanen H, Adamsson A. Cryptorchidism and endocrine disrupting chemicals. Mol Cell Endocrinol. 2012;355:208-20.

4. Brain C, Creighton SM, Mushtaq I, et al. Holistic management of DSD. Best Pract Res Clin Endocrinol Metab. 2014;24:335-54.

5. El-Sherbiny M. Disorders of sexual differentiation: I. Genetics and pathology. Arab J Urol. 2013;11:19-26.

6. Shalaby M, Shoma A, Elanany F, Elganainy E, El-Akkad MA. Management of the looping vas deferens during laparoscopic orchiopexy. J Urol. 2011;185 Suppl 6:2455-7.

7. Gatti JM, Ostlie DJ. The use of laparoscopy in the management of nonpalpable undescended testes. Curr Opin Pediatr. 2008;19:349-53.

8. Riquelme A. Orquidopexia laparoscópica en el testículo no descendido, palpable y no palpable. Rev Mex Urol. 2009;69:215-8.

9. Sheikh A, Mirza B, Ahmad S, et al. Laparoscopic management of 128 undescended testes: Our experience. Afr J Paediatr Surg. 2012;9:106-8.

10. Hrivatakis G, Astfalk W, Schmidt A, et al. The Timing of surgery for undescended testis. A retrospective multicenter analysis. Dtsch Arztebl Int. 2014;111:649-57.

11. Alemayehu H. Cryptorchid testis e. An unusual intra-abdominal location. J Ped Surg Case Reports. 2013;1:401-2. 